\title{
Heavy quarks and long-lived hadrons
}

\author{
Thomas Appelquist* \\ Institute for Advanced Study, Princeton, New Jersey 08540 \\ H. David Politzer ${ }^{\dagger}$ \\ California Institute of Technology, Pasadena, California 91125 \\ (Received 14 April 1975)
}

\begin{abstract}
In a recent Letter we reported on some work which led us to suggest the possibility of narrow spikes in the $e^{+} e^{-}$annihilation cross section into hadrons. In this paper, we discuss the theoretical infrastructure of this work more thoroughly, and improve and extend the calculations and experimental predictions. We examine a colored quark-gluon model of hadronic matter, with color an exact SU(3) gauge symmetry. In addition to the light quarks that make up ordinary hadrons, a heavy quark, such as the charmed $c$, is included. The narrow resonances recently discovered by the MIT-BNL and SLAC-LBL groups are interpreted as $c \bar{c}$ bound states (orthocharmonium). In this energy range, the effective coupling has become small according to asymptotic freedom, and many aspects of the bound-state structure can be calculated. The existence of $0^{-}$ (paracharmonium) states is predicted, and decay widths and mass splittings are estimated. The total $e^{+} e^{-}$ cross section into hadrons is predicted to scale asymptotically, with an approach to scaling from above that can be calculated over a large energy range.
\end{abstract}

\section{INTRODUCTION}

Two groups (MIT-BNL ${ }^{1}$ and SLAC-L $\mathrm{BL}^{2,3}$ ) have recently announced the discovery of new heavy, long-lived particles. More recently, the $e^{+} e^{-}$ cross section has been measured ${ }^{4}$ up to $E_{\text {c.m. }}$. $=5 \mathrm{GeV}$ and an enhancement discovered in the vicinity of $4.1 \mathrm{GeV}$. We discuss here some recent theoretical work ${ }^{5}$ which was stimulated by the large $e^{+} e^{-}$cross section reported some time ago ${ }^{6}$ and which has predictive impact on the new developments. It is fundamentally an analysis of the $e^{+} e^{-}$total annihilation cross section into hadrons in an asymptotically free quark-gluon theory, although more general consequences of the model will emerge. The failure of the total cross section to scale $^{6}$ led us to consider the possibility ${ }^{7}$ that in addition to the light quarks that make up ordinary hadrons, there exists a heavy quark such as the charmed $c$ with $2 m_{c} \approx 3 \mathrm{GeV}$.

We examine a colored-quartet model with color an exact SU(3) gauge symmetry. If the asymptotic freedom of this model is to explain Bjorken scaling, ${ }^{8}$ then at momentum scales above one or two $\mathrm{GeV}$, the effective coupling strength must have become small. This means that perturbation theory can be applied at energies of order $2 m_{c}$; however, approximate scale invariance will emerge only for energies substantially higher than $2 m_{c}$. $^{9}$

We analyze the $e^{+} e^{-}$total annihilation cross section into hadrons in perturbation theory. At energies above the nominal two-quark threshold $2 m_{c}$, the perturbation expansion is well behaved, and we can calculate the deviation from scale invariance due to the finiteness of $m_{c}$. In a domain about $2 m_{c}$, however, the expansion breaks down as small internal momenta dominate the Feynman integrals. This produces two kinds of breakdown, the first coming from Coulomb-like singularities associated with massless particle exchange.

These can be handled just as in electrodynamics, by first summing uncrossed ladders and then perturbing about that. The result is a Balmer series of resonance peaks in the total cross section with spacing and widths calculable in perturbation theory. However, a second kind of breakdown due to "infrared slavery" destroys this simple picture. The internal momenta are small enough so that Yang-Mills-type radiative corrections lead to a large effective coupling strength associated with the binding. Nevertheless, we shall see that many features of the resonance structure can be calculated.

In Sec. II, we define and motivate the model of strong interactions to be examined. We discuss the renormalization of the theory and the effective coupling strength as a function of the momentum scale. Particular attention is paid to the breakdown of the perturbation expansion for $\sigma\left(e^{+} e^{-}\right.$ $\rightarrow$ hadrons) due to the presence of quark thresholds.

Section III is devoted to the perturbative analysis of the total $e^{+} e^{-}$annihilation cross section above and below $2 m_{c}$. The total cross section is argued to scale for $E_{\text {c.m. }} \gg 2 m_{c}$ with a calculable approach to scaling. The perturbation expansion is shown to break down in a domain about $2 m_{c}$ because of Coulomb-like singularities. A partial summation of the perturbation expansion suggests $c \bar{c}$ bound 
states. However, this particular approximation proves unsatisfactory because a positroniumlike picture of these states is shown to be inconsistent, because of the importance of Yang-Mills infrared effects at the typical momentum transfers involved. We discuss what can and cannot be computed in perturbation theory. The predicted qualitative structure of $\sigma_{e^{+} e^{-} \rightarrow \text { hadrons }}\left(E_{\text {c.m. }}\right)$ from $E_{\mathrm{cm} \text {. }}$. $\sim 1 \mathrm{GeV}$ to $E_{\text {c.m. }} \gg 2 m_{c}$ is summarized.

In Sec. IV, we present calculations and experimental predictions. Properties of the observed orthocharmonium states and predicted paracharmonium states are computed. Using these results, we estimate the approach to asymptotic scaling from $E_{\text {c.m. }} \sim 5 \mathrm{GeV}$ through SPEAR II and higher energies. We compare charmonium dynamics with that of the conventional mesons such as the $\phi$ and $\eta^{\prime}$.

In Sec. V, we discuss the problem of higher light-quark thresholds. The charmonium states decay into ordinary hadrons through vector gluons. We discuss the possible existence of gluonic matter (quarkless resonances) in these decays.

\section{PRELIMINARIES}

\section{The model}

The strong-interaction model to be analyzed is defined by the Lagrangian density

$$
\mathcal{L}=-\frac{1}{4} F_{\mu \nu}^{a} F^{a \mu \nu}+\bar{\psi}\left(i \not D-m_{0}\right) \psi .
$$

$\psi$ is a set of twelve quark fields describing a quartet of quarks $u, d, s$, and $c$ each coming in three colors: red, white, and blue. ${ }^{10}$ Each color multiplet has a single mass, and color is taken to be an exact SU(3) gauge symmetry. Thus, there is no vector field mass term. $F_{\mu \nu}^{a}$ is the gauge-covariant curl, and $D_{\mu}$ is the covariant derivative:

$$
\left(D_{\mu} \psi\right)_{n}=\partial_{\mu} \psi_{n}-\frac{1}{2} g A_{\mu}^{a}\left(\lambda^{a}\right)_{n m} \psi_{m},
$$

where $\psi_{m}$ is one of the four color triplets. The symmetries of the theory [the breaking of $U(4)$ $\times U(4)]$ are just the symmetries of the bare-quark mass matrix $m_{0}$. We will take $m_{c} \gg m_{u}, m_{d}, m_{s}$.

For our purposes, the most important feature of this model is its asymptotic freedom, ${ }^{8}$ which underlies our entire perturbative analysis. The accompanying infrared instability may be the source of the strong binding forces that apparently allow only color-singlet physical states. We emphasize that the main thrust of this work is dynamical: an examination of a theory that has both asymptotic freedom and a heavy quark. Although we use the SU(4) model with its heavy, charmed quark of neutral $\Delta s=1$ fame, this is not essential. The numerical results of Sec. IV will depend on the details of the model but modifications appropriate to other heavy-quark models can easily be made.

\section{Renormalization}

We define the renormalized coupling constant $g$ in terms of two- and three-point functions at some Euclidean momentum configuration of scale $M$. A convenient choice is the vector-meson propagator and the 1PI three-vector-meson vertex at a symmetric point. ${ }^{11}$ The asymptotic freedom of the model assures us that with $M$ taken large enough, $\alpha_{s} \equiv g^{2} / 4 \pi$ will be arbitrarily small. If Bjorken scaling is to be explained by this kind of theory, then $\alpha_{s} \ll 1$ for $M \geqslant 1-2 \mathrm{GeV}$, a condition easily satisfied by the numerical fits of Sec. IV.

The choice of a particular definition of renormalized quark mass is a matter of convenience. Any two variants, $m$ and $m^{\prime}$, are related perturbatively by $m^{\prime}=m\left[1+O\left(\alpha_{s}\right)\right]$. A brief discussion of our choice and related ones is given below.

If the Lagrangian (2.1) describes the strong interactions, the quark mass matrix characterizes the first level of hadronic symmetry breaking. ${ }^{12}$ A standard analysis begins with the transformation properties of the symmetry-breaking term in the Hamiltonian. ${ }^{13}$ Hence, it seems desirable to have the renormalized mass matrix proportional to the bare mass matrix, e.g., $m_{i}=Z m_{0 i}$ with a common $Z$. (Note that a common mass renormalization is sufficient to render all Green's functions finite because the logarithmic cutoff dependence of each $m_{0 t}$ is mass independent.)

For our purposes, it will be convenient to adjust $Z$ so that to any finite order of perturbation theory, the $c$ propagator has a pole at $\not p=m_{c}$. This imitates the conventional prescription in electrodynamics and facilitates the translation of various electrodynamic calculations into the present context. Note that this perturbative prescription leaves open the true structure of the $c$ propagator near $\not p=m_{c}$. This depends on the inf rared behavior of the theory, ${ }^{14}$ which is necessarily a strong-coupling problem in an asymptotically free theory, regardless of what is arranged in perturbation theory. In particular, it is an open question whether quarks can exist as physical particles. With $Z$ fixed by an "on-shell" subtraction of the $c$, we do not have the freedom of also subtracting the light quarks on shell. Thus, the inverse $u$ quark propagator will not vanish at $\not p=m_{u}$, but this is not important.

Another choice of $Z$, which is convenient from the standpoint of the renormalization-group equation, is to make all renormalizations independent of the renormalized masses. ${ }^{15,16} \mathrm{~A}$ particular 
example is suggested in Ref. 15: All wave-function and charge renormalizations are performed with $m_{i}=0$, at momenta characterized by a scale $M$. The mass divergences of the $m_{i} \neq 0$ theory are removed by identifying $m_{i}$ by $m_{i}=Z^{-1} m_{0 i}$ where $Z$ renormalizes the operator $\bar{\psi} \psi$ in the $m_{i}=0$ theory. We will not make explicit use of this prescription, but it will be mentioned again in discussion of the renormalization-group formalism that underlies the calculations.

\section{A bound on the integrated $e^{+} e^{-}$total cross section}

The application of this model to $e^{+} e^{-}$annihilation into hadrons requires a certain amount of care. The most direct and reliable (but probably least exciting) thing to be done is to place a bound on the integrated total cross section. The hadronic vacuum polarization $\pi\left(q^{2}\right)$ with spacelike $q^{2}$ is given, through its spectral form, by such an integral. Asymptotic freedom allows a perturbative calculation ${ }^{17}$ of $\pi\left(q^{2}\right)$ for $-q^{2} \gtrless 1 \mathrm{GeV}^{2}$, and thus one can bound the integrated total cross section. This has been looked at in detail by Adler ${ }^{18}$ for a three-triplet model and can easily be extended to the three-quartet model taking into account the effect of the heavy-quark mass in the perturbative calculation.

$$
\begin{gathered}
\sigma_{\text {tot }}\left(e^{+} e^{-} \rightarrow \text { hadrons }\right) \text {, perturbation theory, and the } \\
\text { renormalization group }
\end{gathered}
$$

Much of what we do from here on involves the use of perturbation theory for timelike $q^{2}$. This section is devoted to providing a partial justification for this and to pointing out the problems. In the case of an asymptotically free theory, the question is simply whether or not the perturbation expansion breaks down. The renormalization mass $M$ is chosen large enough so that $\alpha_{s} \equiv g^{2} / 4 \pi$ $\ll 1$, and perturbation theory can be used providing dynamical effects (such as large logarithms) do not enter which make the effective expansion parameter large. We must show that successive terms of the expansion in powers of $\alpha_{s}$, in fact, get systematically smaller for the kinematic domain of interest.

First suppose there are no mass parameters in the Lagrangian. Euclidean Green's functions still exist since the external momenta provide an infrared cutoff. This follows from the analysis of Kinoshita. ${ }^{19}$ If all the external momenta are on the order of $M$, there will be no large logarithms to destroy the perturbation expansion. The Kinoshita theorem can also be applied to a timelike Green's function such as the hadronic vacuum polarization in this model. Its imaginary part is proportional to the total hadronic cross section, and the Kinoshita theorem tells us that $\sigma_{\text {tot }}$ is finite in each order for nonzero $s$ (the center-ofmass energy squared) in this zero-mass theory. Hence, the coefficients of $\alpha_{s}^{n}$ in the expansion for $\sigma_{\text {tot }}$ are all finite.

We expect these coefficients to be of comparable magnitude as long as $s$ is of the order of $M^{2}$, and typically they contain powers of $\log \left(s / M^{2}\right)$. For very large or small $s / M^{2}$, we can use the renormalization-group apparatus to improve the ailing perturbation expansion. This amounts to recognizing that the coupling constant $g$ is actually a function of the renormalization mass $M: g$ $=g(M)$. We are free to choose an $M$ appropriate to a given $s$, e.g., choose $M=\sqrt{s}$. Thus, for the object $R(s) \equiv\left(3 s / 4 \pi \alpha^{2}\right) \sigma\left(e^{+} e^{-}-\right.$hadrons $)$,

$$
R\left(\left(s / M^{2}\right), g(M)\right)=R(1, g(\sqrt{s})),
$$

and there are no large logarithms in the expansion of the right-hand side in powers of $g(\sqrt{s})$ for any $s .^{20}$ Of course, now the question is the size of $g(\sqrt{s})$. We infer from asymptotic freedom and Bjorken scaling that $g(\sqrt{s})$ is small for $\sqrt{s} \approx 1-2$ $\mathrm{GeV}$, decreases like $1 / \log s$ for larger $s$, and increases to a nonperturbative value below $1 \mathrm{GeV}$.

The introduction of nonzero quark masses $m_{i}$ offers no serious complication in the renormalization-group analysis. For simplicity we consider the definition of $m_{t}$ given earlier for which all renormalization constants are $m_{i}$ independent Just like $g, m_{i}$ will also depend implicitly on $M$ and we can construct the function $m_{i}=m_{i}(M)$. [The virtue of these renormalization conventions is that $g(M)$ behaves as in the massless theory and is independent of $m_{i}$.] Now we can write

$$
R\left(\frac{s}{M^{2}}, \frac{m_{i}(M)}{M}, g(M)\right)=R\left(1, \frac{m_{i}(\sqrt{s})}{\sqrt{s}}, g(\sqrt{s})\right) .
$$

For a quark-gluon theory, $m_{i}(\sqrt{s})$ can be computed for any $s$ such that $g(\sqrt{s})$ is small; in that domain, $m_{i}(\sqrt{s})$ increases as a positive power of logs. Any other definition of $m_{i}$ may differ in a calculable fashion that is higher order in $g$.

If we are interested in the behavior of $\sigma_{\text {tot }}$ for a modest range of $s$, Eq. (2.4) is really no improvement. We can just as well choose $M$ to lie within that range and use ordinary perturbation theory. The difficult problem is the application of perturbation theory in the timelike region for nonzero quark masses. As $s$ increases, no matter how large it becomes, it will always be passing through multiples of the quark masses - the nominal quark thresholds. This introduces a new small momentum, typically occurring in denomin- 
ators and logarithms to arbitrarily high powers in higher and higher orders of the expansion. Thus, perturbation theory may be unreliable. This does not occur for spacelike external momenta for the vacuum polarization, for example, because one is never near a threshold, and Feynman integrals are always finite.

We will examine in some detail the perturbative expansion for $\sigma_{\text {tot }}$ near the two-quark threshold and show how useful physical information can be extracted. We will return to the question of multiple quark thresholds in Sec. VI. Our discussion is qualitative and by no means definitive. However, it appears that these thresholds must be unimportant if a simple quark model for $\sigma_{\text {tot }}$ is to be useful.

\section{PERTURBATION THEORY AND ITS BREAKDOWN NEAR $E_{\mathrm{t} . \mathrm{m} .}=2 m_{c}$}

The perturbation expansion for $R(s) \equiv\left(3 s / 4 \pi \alpha^{2}\right)$ $\times \sigma\left(e^{+} e^{-} \rightarrow\right.$ hadrons $)$ begins with the graphs of Fig. 1. The zeroth-order contribution [Fig. 1(a)] is

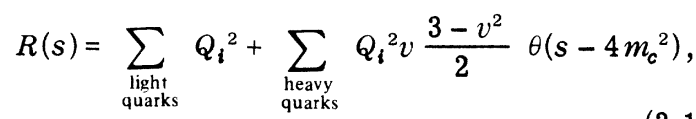

where $v=\left(1-4 m_{c}{ }^{2} / s\right)^{1 / 2}$. We have scaled the lightquark masses to zero, $v$ is the velocity of the $c$ in the c.m. frame, and the kinematic factor in the heavy-quark term is just two-particle phase space. The two sums over quark charges squared are 2 and $\frac{4}{3}$, respectively, in the three-quartet model. This contribution to $R(s)$ is indicated by the dotted line in Fig. 2.

The $O\left(\alpha_{s}\right)$ contribution [Figs. 1(b) and 1(c)] can also be written down immediately by making use of identical electrodynamic calculations. ${ }^{21,22}$ Through this order, we have

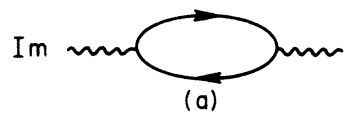

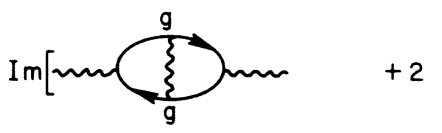

(b)

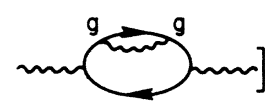

(c)
FIG. 1. (a) Zeroth-order contribution to $\sigma_{\text {tot }}\left(e^{+} e^{-}\right.$ $\rightarrow$ hadrons); (b) and (c) the $O\left(\alpha_{s}\right)$ contributions to $\sigma_{\text {tot }}\left(e^{+} e^{-} \rightarrow\right.$ hadrons $)$.

$$
\begin{aligned}
R(s)= & \sum_{\substack{\text { light } \\
\text { quarks }}}{Q_{i}}^{2}\left(1+a \frac{3 \alpha_{s}}{4 \pi}\right) \\
& +\sum_{\substack{\text { heavy } \\
\text { quarks }}} Q_{i}{ }^{2} \theta\left(s-4 m_{c}^{2}\right) v \frac{3-v^{2}}{2}\left[1+a \alpha_{s} f(v)\right],
\end{aligned}
$$

where $a=\frac{4}{3}$ for an SU(3) color group. The function $f(v)$ is rather complicated (Ref. 22, Eq. 5-4.200) involving a combination of Spence functions, but it approaches $3 / 4 \pi$ asymptotically, in agreement with the light-quark result. Schwinger has written down ${ }^{22}$ an interpolating formula for $f(v)$ which has the correct $v \rightarrow 1$ and $v \rightarrow 0$ behavior and which agrees with the exact $f(v)$ to $\pm 1 \%$ throughout the interval 0 to 1 . This is completely adequate for our purposes; it is

$$
f(v)=\frac{\pi}{2 v}-\frac{3+v}{4}\left(\frac{\pi}{2}-\frac{3}{4 \pi}\right) .
$$

$R(s)$ through order $\alpha_{s}$ is indicated by the solid line in Fig. 2.

As $v \rightarrow 0, f(v) \propto 1 / v$. This behavior comes from Fig. 1(b) and is a consequence of a Coulomb-like final-state interaction. In $n$th order, $n$ massless vector exchanges (uncrossed ladders) give $n$ factors of $1 / v$. This breakdown of the perturbation expansion for small $v$ is connected to a breakdown below $E_{\text {c.m. }}=2 m_{c}$. There, the sum of (uncrossed) gluon exchanges is responsible for the formation of positroniumlike bound states. Three important comments about this behavior are in order.

(1) For $E_{\text {c.m. }}$ large enough so that $\frac{4}{3} \alpha_{s} f(v) \ll 1$, second-order perturbation theory will suffice to

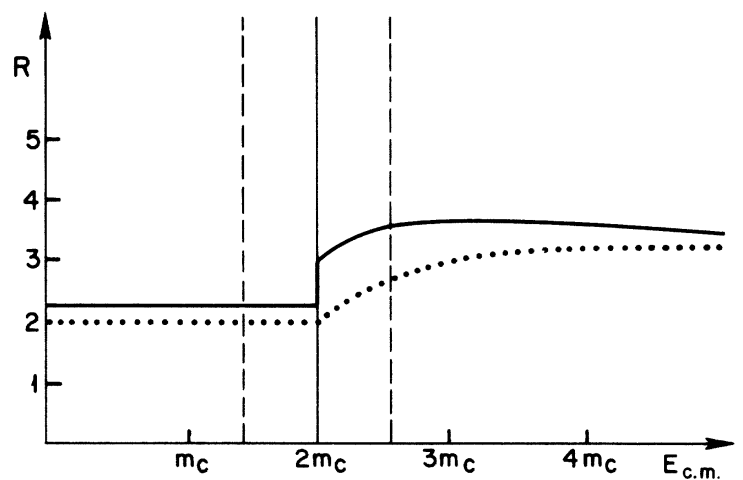

FIG. 2. $\boldsymbol{R}\left(\boldsymbol{E}_{\text {c.m. }}\right)$ through order $\alpha_{s}$ in the three-quartet model. The dotted line is the zeroth-order contribution which approaches $\frac{10}{3}$ asymptotically. The solid line is the sum of the zeroth- and first-order terms for $\alpha_{s}=0.2$. The perturbation expansion becomes unreliable for $E_{\text {c.m. }}<1 \mathrm{GeV}$ and within a $2-\mathrm{GeV}$ region centered on $E_{\text {c.m. }}=2 m_{c}$. For $m_{c} \approx 2 \mathrm{GeV}$, this region is indicated by the vertical dashed lines. 
calculate the approach to free-quark-model scaling. Numerical estimates of this approach are presented in Sec. IV. An important point of princi$p l e$ is that the observation of this drop in $R$ would provide a measurement of the parameter $m_{c}$. This mass parameter, defined according to the renormalization prescription in Sec. II, is measurable despite the possible permanent confinement of the quark.

(2) In the nonrelativistic limit, $v \rightarrow 0$, the sum of all the uncrossed ladder exchanges between the $c$ quarks can be summed exactly. In this limit, $1+\frac{4}{3} \alpha_{s} f(v)-1+\frac{4}{3} \alpha_{s}(\pi / 2 v)$, which are the first two terms in the expansion of the nonrelativistic enhancement factor

$$
\frac{\left|\psi_{\text {Coulomb }}(0)\right|^{2}}{\left|\psi_{\text {Coulomb }}(\infty)\right|^{2}}=\frac{\frac{4}{3} \alpha_{s} \pi / v}{1-\exp \left(\frac{4}{3} \alpha_{s} \pi / v\right)} .
$$

(3) The breakdown of the perturbation expansion near threshold due to Coulomb singularities is thus easily dealt with. There is, however, another more serious breakdown coming from the nonAbelian structure of the theory. As threshold is approached, the typical momentum transfer flowing through exchanged gluons decreases. This means that the higher-order corrections to these lines become more and more important, increasing the effective coupling strength. A measure of the typical momentum transfer $\langle k\rangle$ is $\left\langle k^{2}\right\rangle$ $\sim \frac{1}{4}\left(E_{\text {c.m. }}{ }^{2}-4 m_{c}{ }^{2}\right)$ and when this is less than about $1 \mathrm{GeV}^{2}$, the effective coupling strength will have become large (order 1).

Below $E_{\text {c.m. }}=2 m_{c}$, the effect of the $c$ is first seen in order $\alpha_{s}{ }^{3}$, e.g., Fig. 3, where the only Cutkosky cut is through the three gluons. This graph and similar higher-order graphs remain small corrections to the dominant light-quark graphs of Fig. 1 until $\left(4 m_{c}^{2} / s-1\right)^{1 / 2} \sim \alpha_{s}$. In this region one again encounters both Coulombic and Yang-Mills-infrared breakdown of the perturbation expansion. The Coulombic ladder exchanges between the quarks of Fig. 3 produce positroniumlike narrow resonances, orthoharmonia. If uncrossed ladders were really the dominant contribution in this region, then the charmonium system would be a positronium duplicate with a Balmer series of resonances whose widths are given by three-gluon decay.

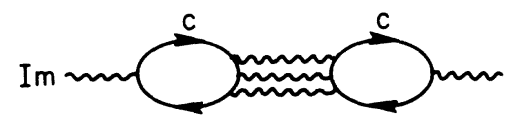

FIG. 3. Lowest-order perturbative contribution involving heavy-quark loops below $E_{\text {c.m. }}=2 m_{c}$.
The Yang-Mills breakdown will obliterate this lovely picture. The typical momentum transfer in an exchanged gluon is $\langle k\rangle \sim\left(m_{c}{ }^{2}-E_{\text {c.m. }}{ }^{2} / 4\right)^{1 / 2}$. In a positronium picture, the ground state is at $E_{\text {c.m. }}=2 m_{c}-\frac{1}{4}\left(\frac{4}{3} x_{s}\right)^{2} m_{c}$, which gives $\langle k\rangle$ $\sim \frac{1}{2}\left(\frac{4}{3} \alpha_{s}\right) m_{c}$, the inverse Bohr radius. For $\alpha_{s} \leqslant 0.3$ and $m_{c} \leqslant 2 \mathrm{GeV},\langle k\rangle \leqslant 300 \mathrm{MeV}$, and the YangMills corrections will certainly have become important. In fact, the Yang-Mills corrections will begin producing a large effective coupling strength as soon as $\langle k\rangle \lesssim 1 \mathrm{GeV}$ corresponding to $E_{\mathrm{c} . \mathrm{m}}$. $\gtrsim 2 m_{c}-1 \mathrm{GeV}$. Altogether the re will be a domain of width $\sim 2 \mathrm{GeV}$ centered on $E_{\text {c.m. }}=2 m_{c}$ in which the theory becomes strongly coupled because of the infrared instability of the Yang-Mills theory. This domain is indicated in Fig. 2.

If the spikes at $E_{\text {c.m. }}=3.1$ and $E_{\text {c.m. }}=3.7$ are bound states in this model, they will certainly lie inside the strong-coupling domain. Thus we do not expect a Balmer series, and, in fact, a simple Coulomb picture will not even describe the ground state. Nevertheless, some things can be calculated in perturbation theory. Even though the effective coupling strength responsible for $c \bar{c}$ binding has become large and the Coulombic picture broken down, the coupling strength associated with the annihilation into states containing no $c$ quarks remains weak. The relevant momentum scale here is $E_{\text {c.m. }}$, not the momentum transfer, and this lies in the weak-coupling regime. As shown in Fig. 4, we divide the transition of two $c$ quarks to ordinary hadronic matter into a part A which represents binding, i.e., A contains all possible interactions of the $c$ 's, and a part $B$, representing their annihilation into other quarks and gluons. Thus, B is defined by being two-charmedquark irreducible. As the $c$ and $\bar{c}$ approach their mass shells, A contains severe infrared divergences in perturbation theory, indicating that the expansion is no good; it must be reexpressed in terms of a larger effective expansion parameter. However, B is regular in each order, so we can expect successive orders in perturbation theory to actually get smaller by powers of $\alpha_{s}$ (as defined by the scale of the problem, $E_{\text {c.m. }}$.). The possible effects of very light other quarks on this argument are closely related to the general problem of multiple-quark thresholds. (See Sec. V.)

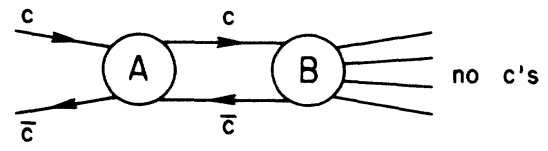

FIG. 4. The transition of a $c \bar{c}$ pair into ordinary hadronic matter (uncharmed quarks and gluons). 
Applying ordinary perturbation theory to $\mathrm{B}$, we can ask which final states correspond to the largest amplitudes. The hadronic state occurring first, which contains no $c$ quarks, is three gluons, as indicated in Fig. 5(a) (three because we are interested in a color singlet and because of charge conjugation). Our ignorance of the binding mechanism or quark wave function can be canceled out by comparing the total ordinary hadronic decay to the electromagnetic decay indicated in Fig. 5(b). This observation is the basis of the calculations of the next section.

Let us return briefly to the question of a Coulomb-type approximation of the binding. It breaks down for the $c$ quark but if there are other yet heavier quarks, Coulomb wave functions may be accurate descriptions of at least the lowestlying states. (Note that as the quarks get heavier, they tend to remain closer together, and at short distances single-gluon exchange certainly does accurately describe the interaction.) Thus for an $8 \mathrm{GeV}$ quark, for example, we have a reliable description of the binding of $q$ and $\bar{q}$ and can estimate any decay of such a hadron. (The excited states would be strongly bound, however, and certainly smaller in extent than indicated by the Bohr model.)

Attempts have been made to treat the dynamics of the strong-coupling region. Even though the Coulombic picture breaks down for the 3.1 and 3.7 resonances, it is very likely that the motion of the $c$ and $\bar{c}$ is nonrelativistic. Several groups ${ }^{23,24}$ have assumed this and solved a Schrödinger equation with a linearly rising potential to represent Coulombic breakdown at large distances and quark confinement. With a potential containing both a short-distance Coulombic piece and a linear piece, ${ }^{23}$ it is found that the linear piece dominates for both the 3.1 and 3.7 and that the motion is indeed nonrelativistic, justifying use of the Schrödinger equation. It is clearly an extremely important problem to elucidate the connection of this approach to the underlying field theory. This comment may not be as specious as it sounds. The nonrelativistic character of the charmonium system means that a Bethe-Salpeter formalism

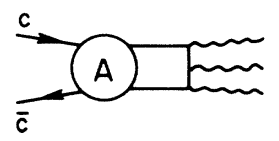

(a)

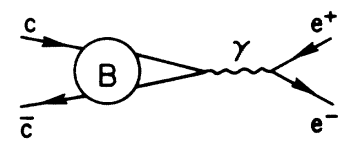

(b)
FIG. 5. (a) The transition of a $c \bar{c}$ pair into three gluons; (b) the electromagnetic transition of a $c \bar{c}$ pair into $e^{+} e^{-}$. may be useful in the sense that the skeleton expansion of the kernel could be truncated. The single ladder exchange with vertex and self-energy insertions might give the Schrödinger equation, and relativistic and spin corrections could be calculated.

\section{CHARMONIUM PHENOMENOLOGY AND NUMERICAL ESTIMATES}

In this section, we tabulate some of the numerical predictions appropriate to the colored $\mathrm{SU}(4)$ quartet model. These are improvements and extensions of the estimates in Ref. 6 and focus mainly on perturbative calculations and the structure of the $e^{+} e^{-}$total annihilation cross section. A more extensive discussion of the charmonium level structure and decay modes has been given by Appelquist et $a l .^{25}$ and by Eichten et $a l .^{23}$ For a more complete treatment of charm phenomenology, we refer the reader to the review of Gaillard, Lee, and Rosner. ${ }^{26}$ We emphasize again that these calculations can easily be modified to describe'any asymptotically free model with heavy quarks.

\section{Properties of the 3.1}

We have argued that perturbation theory can be applied to the two-c-irreducible matrix element for the process $c \bar{c}-$ states containing no charmed quarks (Fig. 4). The lowest-order contribution is the transition to three gluons shown in Fig. 5(a). Even though the perturbation expansion for the binding breaks down because of non-Abelian infrared structure, the motion is nonrelativistic and the radius of the $c \bar{c}$ bound state is large compared to the $c$ Compton wavelength. ${ }^{23}$ Thus to leading order in $v / c$,

$$
\Gamma(3.1-\text { hadrons })=\left|M_{h}(3.1)\right|^{2}|\psi(0)|^{2},
$$

where for $M_{h}$ we take the spin-summed and colorsummed matrix element into three gluon $\mathrm{s}^{27}$ :

$$
\left|M_{h}(3.1)\right|^{2}=\frac{16}{9 \pi}\left(\pi^{2}-9\right) \frac{5}{18} \alpha_{s}^{3} \frac{1}{m_{c}^{2}} .
$$

The wave function at the origin enters since the annihilation takes place in a region of order $1 / m_{c}$ in radius.

The leptonic width via one photon into $l l$ is

$$
\Gamma(3.1-\tau l)=\left|M_{l}(3.1)\right|^{2}|\psi(0)|^{2},
$$

where

$$
\left|M_{l}(3.1)\right|^{2}=4\left(Q_{c} \alpha\right)^{2} m_{c}^{2} .
$$

In the ratio, the unknown wave function cancels, and we have 


$$
\frac{\Gamma(3.1-\tau l)}{\Gamma(3.1-\text { hadrons })}=\frac{\frac{9}{4} \pi\left(Q_{c} \alpha\right)^{2}}{\left(\pi^{2}-9\right) \frac{5}{18} \alpha_{s}^{3}} .
$$

If we take $\Gamma(3.1 \rightarrow \tau l)=5.2 \mathrm{keV}, \Gamma(3.1 \rightarrow$ hadrons $)$ $=55 \mathrm{keV},{ }^{28}$ and $Q_{c}=\frac{2}{3}$, we find

$$
\alpha_{s}=0.2 \text {. }
$$

This value of $\alpha_{s}$ along with $m_{c} \leqslant 2 \mathrm{GeV}$ means that the momentum transfer in the bound state is too small to allow a Coulombic description of the binding.

Properties of the 3.7

If this peak is interpreted as the first $s$-wave radial excitation of the 3.1 , formulas analogous to (4.1) and (4.3) describe its direct decay into uncharmed hadrons and its leptonic decay. Its leptonic width has been reported to be $\Gamma(3.7-\tau l)$ $=2.5 \mathrm{keV} \cdot{ }^{26,28}$ This is smaller than the 3.1 leptonic width since $|\psi(0)|$ is smaller for the radially excited state. Since the Coulombic description has broken down, quantities like $|\psi(0)|$ and the excitation energy cannot be calculated in perturbation theory. With the linear potential model for $c$ confinement, ${ }^{23,24}$ however, the experimental orders of magnitude seem reasonable. The bulk of the large width $(\sim 0.5 \mathrm{MeV})$ of the 3.7 is presumably due to strong and electromagnetic transitions to lower-lying charmonium states and these have been listed and estimated in Refs. 24 and 26. The one quantity that can be calculated perturbatively ${ }^{27}$ is the ratio of the leptonic width to the direct decay width into uncharmed hadrons. Using the experimental lepton width, this hadronic width is found to be about $50 \mathrm{keV}$, a small fraction of the total width.

Predicted charmonium states

The expected states have been tabulated and discussed in Refs. 23, 25, and 26. We will discuss only the $0^{-}$para states that should accompany both the 3.1 and 3.7. For these states, called $\eta_{c}$ and $\eta_{c}^{\prime}$ in Ref. 27, several important predictions can be made using perturbation theory in $\alpha_{s}$.

The $\eta_{c}$ is expected to sit about $50-100 \mathrm{MeV}$ below the 3.1. A precise calculation of the hyperfine splitting is difficult because of the non-Coulombic structure of the binding. Various estimates are discussed in Ref. 26 and they tend to give the same order of magnitude. The $\eta_{c}$ can decay into hadrons predominantly through two gluons or it can decay electromagnetically into two photons. These widths can be calculated since $|\psi(0)|$ is known from, say, the leptonic width of the 3.1. We find

$$
\begin{aligned}
\Gamma\left(\eta_{c} \rightarrow \text { hadrons }\right) & =\frac{3}{2} \frac{\alpha_{s}^{2}}{\alpha^{2}} \Gamma(3.1-\tau l) \\
& =6.7 \mathrm{MeV}
\end{aligned}
$$

and

$$
\Gamma\left(\eta_{c}-\gamma \gamma\right)=\frac{4}{3} \Gamma(3.1-\tau l)=7 \mathrm{keV},
$$

where we have used $\alpha_{s}=0.2$ and $\Gamma(3.1-\tau l)=5.2$ keV. ${ }^{28}$ Thus the $\eta_{c}$ is expected to be much broader than the 3.1 and to have an electromagnetic branching ratio of $0.1 \%$.

These values lead to a substantial Primakoff production cross section for the $\eta_{c}$. On $\mathrm{Pb}$ with $E_{\gamma}=200 \mathrm{GeV}$, Dashen et al. find ${ }^{29}$

$$
\sigma_{\mathrm{Pb}}\left(\gamma \rightarrow \eta_{c}\right)=100 \mathrm{nb} .
$$

Other ways of finding $\eta_{c}$ include hadronic production and electromagnetic decay of higher charmonium states. See Refs. 23, 25, and 26.

The approach to the asymptotic region

If there are no more heavy quarks, then $R(s)$ should decrease to asymptotic flatness from around $E_{\mathrm{c.m} .}=5 \mathrm{GeV}$. The second-order expression for $R(s)$ is given in Eq. (3.2) and we now know that $\alpha_{s} \approx 0.2$. This coupling constant has been defined at a Euclidean momentum scale $M$ which we take to be $3 \mathrm{GeV}$. The falloff comes from two sources. The first is the decrease of the threshold enhancement near $s=4 m_{c}{ }^{2}$. The second is the logarithmic decrease of the effective coupling constant $\alpha_{s}(s)$ as $s$ increases throughout the region $s \geqslant 1 \mathrm{GeV}^{2}$. For the case of the conventional charm model, we can make some numerical estimates. The transitional domain in which the theory becomes strongly coupled is about $2 \mathrm{GeV}$ in width, extending from $E_{\text {c.m. }} \approx 3 \mathrm{GeV}$ to $E_{\text {c.m. }} \approx 5 \mathrm{GeV}$ (Fig. 2). Below $3 \mathrm{GeV}$, the perturbation expansion should converge and $R(s)$ will be given, through second order, by the first (light-quark) term in Eq. (3.2). With $\alpha_{s} \approx 0.2, R(s) \approx 2.2$ in this region, quite consistent with experiment. ${ }^{4,28}$ Beyond $E_{\text {c.m. }} \approx 5 \mathrm{GeV}$, perturbation theory should again be applicable. $R(s)$ will be given, through second order, by Eq. (3.2) with $\alpha_{s}$ replaced by the running coupling constant $\bar{\alpha}(s)$ :

$$
\bar{\alpha}(s)=\frac{\alpha_{s}}{1+(25 / 12 \pi) \alpha_{s} \log \left(s / M^{2}\right)} .
$$

The approach to $\frac{10}{3}$ is quite rapid. At $E_{\text {c.m. }}=6 \mathrm{GeV}$, for example, we find $R(s)=3.5 \pm 0.2$ where the estimated error comes from the uncertainty in the value of $\alpha_{s} \equiv \bar{\alpha}\left(M^{2}\right)$ and from the uncalculated higher-order terms. Thus if the colored quartet model is the correct theory of strong interactions, $R(s)$ will have to decrease substantially between 
5 and $6 \mathrm{GeV}$. Beyond $E_{\text {c.m. }}=6 \mathrm{GeV}$, the rate of decrease is very slow and, in fact, $R(s)$ should remain nearly constant through $E_{\text {c.m. }} \approx 9 \mathrm{GeV}$. This results from an interplay between the slowly rising two-particle phase-space factor and the slowly decreasing $\bar{\alpha}(s)$. The accurate measurement of $R(s)$ through these energies is very important for testing the three-quartet model and the specific dynamics of the gauge theory.

\section{Ordinary hadrons}

We have made the point several times that the binding of the $c \bar{c}$ system is predominantly nonCoulombic. If this is true for quarks in the $\mathbf{1 . 5}$ $\mathrm{GeV}$ mass region, it is even more true for the light $u, d$, and $s$ quarks. The spectrum of the conventional hadrons will depend strongly on the long-range quark-confining forces. The dynamics of these systems is further complicated by the fact that, unlike charmonium, the quark motion is relativistic. Other than charmonium, the mesons most treatable by nonrelativistic methods are those with quark content $c \bar{s}$ such as the (predicted) $F^{*}$ with $J^{P}=0^{-}$.

What about the decay-rate calculations? For example, can the ratio $\Gamma(\phi \rightarrow \tau l) / \Gamma(\phi \rightarrow 3 \pi)$ be computed? If the $\phi$ is a pure $s \bar{s}$ state, then the $3 \pi$ decay must go via annihilation into gluons. If the decay can be considered to take place at the origin, that is, if the $\phi$ radius is much bigger than $1 / m_{s}$, then $|\psi(0)|^{2}$ enters both the leptonic and three-gluon decay widths. It cancels in the ratio so that one predicts

$$
\frac{\Gamma(\phi-\tau l)}{\Gamma(\phi \rightarrow 3 \pi)}=\frac{9 \pi \alpha^{2}}{10\left(\pi^{2}-9\right) \alpha_{s}{ }^{3}(1 \mathrm{GeV})} .
$$

With $\alpha_{s}(3.1 \mathrm{GeV}) \approx 0.19$, the renormalizationgroup equation leads to $\alpha_{s}(1 \mathrm{GeV}) \approx 0.26$, so that $\Gamma(\phi \rightarrow \tau l) / \Gamma(\phi \rightarrow 3 \pi) \approx 0.01$. Experimentally, this ratio is $\approx 0.002$, which means we have probably underestimated the nonstrange width by a factor of about five.

There are at least two possible sources of this error. First of all, the effective coupling constant is large enough so that the higher-order terms in the perturbation expansion may not be so small. More importantly, the locality assumption [the use of $|\psi(0)|]$ in the hadronic decay is probably bad. The annihilation takes place over region of order $1 / m_{s}$ is radius and this may not be small compared to the $\phi$ radius. This is certainly an effect that will increase the hadronic width.

A frequently repeated "explanation" for the narrowness of the 3.1 and 3.7 is the OZI rule. ${ }^{30}$ For meson decays, it says that decay amplitudes corresponding to connected quark diagrams (in which $q$ and $\bar{q}$ lines run through the diagram) are allowed and those corresponding to diagrams involving $q \bar{q}$ annihilation or creation are strongly suppressed. For the direct decays of 3.1 and 3.7, the $c \bar{c}$ pair must annihilate and asymptotic freedom offers a possible explanation of this rule. Note, however, that one does not calculate dualitylike diagrams involving initial and final $q \bar{q}$ pairs but rather the decay into gluons [Fig. 5(a)]. For the $\phi$ meson, the coupling constant $\alpha_{s}(1 \mathrm{GeV})$ is probably still small enough so that asymptotic freedom can explain the purity of the $\phi$ as an $s \bar{s}$ state and its narrowness into nonstrange hadrons. ${ }^{31}$ We have seen, however, that the width calculation is more complicated than for the 3.1 and 3.7.

$0^{-}$states can communicate with two gluons so that Zweig's rule should be less operative. Thus, for example, $\eta_{c}$ is expected to be broader than the 3.1 [Eq. (4.7)] and the $\eta^{\prime}$ is much less a pure $s \bar{s}$ state than is the $\phi .^{26}$ The OZI rule seems also to be operative in the decay $3.7 \rightarrow 3.1+\pi \pi$, which involves a disconnected quark diagram. ${ }^{32}$ Here, the momentum flowing into the ordinary hadrons is about $600 \mathrm{MeV}$, which is getting quite small to believe in an asymptotic freedom explanation of OZI suppression.

\section{ADDITIONAL REMARKS}

Higher thresholds

Having made a big issue out of the breakdown of perturbation theory at a two-quark threshold, we must face the question of the behavior at higher thresholds. Even for the interpretation of charmonium, this question is not academic because of the light quarks. Our estimates of $R(s)$ assumed $R_{\text {light quarks }}(s)=2+\theta\left(\alpha_{s}\right)$. Certainly, perturbation theory breaks down at each and every threshold in sufficiently high orders because, even without Yang-Mills corrections, there will be pairwise Coulombic interactions. So for $s \gg m_{i}$, instead of becoming simple, our expansion gives a rapid succession of unreliable threshold singularities.

In what sense can these high threshold effects be unimportant in certain quantities such as $R$ ? First recall that the $(1 / v)^{n^{\prime}}$ singularities, where $v$ is some generalized threshold factor, e.g., $v$ $=\left(1-n^{2} m^{2} / s\right)^{1 / 2}$, are a signal that nonperturbative effects are important. We propose the following interpretation of these singularities. The lowestorder description of any quark threshold (given by the tree graphs for $e^{+} e^{-} \rightarrow n$ quarks), i.e., $g^{2 n-4} \phi_{n}$, where $\phi_{n}$ is the phase space for $n$ quarks, is reliable if smeared over the vicinity of the threshold. That is to say that the integral of the true total cross section in that vicinity exceeds 
the lowest-order description by a correction of order $\alpha_{s}\left(E_{\text {c.m. }}\right)$. (Furthermore, remember $\alpha_{s}\left(E_{\text {c.m. }}\right) \rightarrow 0$ as $E_{\text {c.m. }} \rightarrow \infty$.) Locally, for a particular $E$, the nonperturbative effects may be large when compared to the calculation in any finite order; however, the actual enhancement above the naive expectation (i.e., $\left.\alpha_{s}^{n-2} \varphi_{n}\right)$ are $\theta\left(\alpha_{s}^{n-1}\right)$ when smeared.

This is what would happen in pure QED for $\sigma_{e^{+} e^{-}}$. At high energies, the possibility of producing several positronium atoms or even molecules drastically alters the shape of $\sigma$ near thresholds. But the net effect on a smeared $\sigma$ is negligible. In the quark-gluon theory, the fact of quark binding is signaled by the threshold singularities, while the smeared $R$ may be computable.

We offer only a crude consistency argument, following the dispersive analysis used to bound the integrated cross section near the two-quark threshold. We assume that the nonperturbative effects are confined to the regions where perturbation theory breaks down. Hence, near $s=n^{2} m^{2}$, the contribution of states with fewer than $n$ qua rks (and any number of gluons) is well described perturbatively, i.e., successive orders in $\alpha_{s}$ are, in fact, less and less important. So the production of fewer than $n$ quarks provides a fairly constant and small background near $s=n^{2} m^{2}$. We propose a model for $R$ which consists of the computable background at any $s$ (i.e., fewer than $s^{1 / 2} / m$ quarks in the "final" state) plus the appropriate lowestorder threshold factor for each threshold plus everything else:

$$
R=R_{\text {background }}+R_{\text {threshold }}+\Delta R \text {. }
$$

This is free of content until we approximate $\Delta R$. We assume that $\Delta R$ is, in fact, localized about each threshold and assume further that $\Delta R(s) \geqslant 0$. If we are only interested in smeared quantities, such as $\Delta A_{n}=\int_{n} \Delta R d E$ integrated over the neighborhood of the $n$th threshold, it is sufficient to approximate $\Delta R$ by a sum of $\delta$ functions. If $R$ is bounded as $s \rightarrow \infty$, it is related to $\Pi(t)$, the spacelike vacuum polarization, via the usual once-subtracted dispersion relations. We can now, order by order, estimate the $\Delta A_{n}$, since perturbation theory is good for $\Pi(t)$. In particular, by considering the $\theta\left(\alpha^{n-2}\right)$ calculation, we deduce that $\Delta A_{n}=0+\theta\left(\alpha^{n-1}\right)$.

If one seeks causes for skepticism, they are plentiful. This analysis is inspired by electrodynamics, where the result has been tested, at least crudely, in $e^{+} e^{-}$annihilation. In the quarkgluon theory, the final states are hadrons, so perhaps this expansion is never useful. A more particular criticism might be that the non-Abelian effects are expected to extend $1 \mathrm{GeV}$ above and below each nominal threshold, while the lightquark thresholds are certainly closer than that. Therefore, cumulative effects may be important. We can only point to the flatness of $R$ between 2 and $3 \mathrm{GeV}$ and claim that that is an asymptotic phenomenon.

\section{Gluonic matter}

Assuming the validity of perturbation theory where it is self consistent, we have argued that charmonium decay is well approximated by the decay into three gluons. This requires that these subsequently decay into hadrons with unit probability and that the interactions responsible for hadron production do not significantly alter the gluon production amplitude. What can be said about this hadronic final state? Above some energy, the three gluons are approximately orthogonal to any $q \bar{q}$ state, in that the mixing between them is $\theta\left(\alpha_{s}^{3 / 2}\right)$, and so the expansions of these states in terms of the hadron basis (effectively multipion states) must also be orthogonal.

A theoretical description of the various possibilities for this gluonic matter is given in terms of hypothetical gluon sources $G(x)$ and the source correlation functions. ${ }^{33}$ With the photon treated as an external source of the electromagnetic current $\sum_{i} Q_{i} \bar{\psi}_{i}(x) \gamma_{\mu} \psi_{i}(x)$, we studied $\sigma^{e^{+} e^{-}}$. Similarly, for the $1^{-}$three-gluon source

$$
G_{\mu}(x)=\partial_{\nu}\left[F_{\nu \lambda}^{a}(x) F_{\lambda_{K}}^{b}(x) F_{\kappa \mu}^{c}(x)\right] d^{a b c}
$$

we can study the two-source correlation function, $\left\langle 0\left|G_{\mu}(x) G_{\nu}(y)\right| 0\right\rangle$, or define a ratio $R_{G}$, normalizing the correlation function to the analogous freefield function.

If asymptotic freedom is ever relevant, $R_{G}(s)$ -1 as $s \rightarrow \infty$. Below $1 \mathrm{GeV}^{2}$ or wherever $\alpha_{s}(s)$ is too large for an expansion parameter, we can say nothing except that $R_{G}(s)=0$ for $s<\left(3 m_{\pi}\right)^{2}$, but we can enumerate the possibilities.

But first consider some general features: If $R_{G}(s \rightarrow \infty) \rightarrow 1$, then $R_{G} \approx 1$ for some finite $s$. Following our perturbative philosophy, $R_{G} \approx 1$ for, say, $s \geq 2 \mathrm{GeV}^{2}$ because order by order there are no anomalously large terms in the expansion for $R_{G}$. But how far down in $s$ can we go? We regard $\omega-\phi$ mixing and $\Gamma(\varphi-3 \pi)$ as evidence that $\alpha_{s}(1$ $\mathrm{GeV})$ is in some sense small. On the other hand, we must also account for the existence of the $\rho^{\prime}$ (1600), which is naturally interpreted as a bound state of two light quarks. Recall that we estimated that even for large $s$, perturbation theory may be unreliable for $1 \mathrm{GeV}$ above and below any nominal threshold. So if $m_{p, n} \leqslant 0.5 \mathrm{GeV}$, the $\rho^{\prime}$ is likely at the upper end of such an interval. 
Thus, $R_{G}$ may be very different from 1 for $s$ $<2 \mathrm{GeV}^{2}$.

The various possibilities are as follows.

(1) $R_{G} \approx 0$ for $s<2 \mathrm{GeV}^{2}$. This seems unlikely if in fact $R_{G} \approx 1$ above $2 \mathrm{GeV}^{2}$, particularly as it contrasts markedly with $R_{e^{+} e^{-}}$which averages out to its free-field value for $0 \leqslant s \leqslant 4 \mathrm{GeV}^{2}$.

(2) $R_{G}$ may rise smoothly above $s=9 m_{\pi}^{2}$, per haps overshoot, and come down to 1 from above. This would correspond to nonresonant multipion intermediate states.

(3) $R_{G}$ may have bumps below $2 \mathrm{GeV}^{2}$, much as $R_{e^{+} e^{-}}$is bumpy. This last possibility is exciting because it is simple to distinguish experimentally. The bumps would manifest themselves as SU(3) singlet resonances. Of course, other pure gluon channels could also have resonances. Well below $1 \mathrm{GeV}$, the strength of the gluon-quark coupling implies that these $\mathrm{SU}(3)$ singlet mesons are as strongly coupled to pions as is anything else. Above $1 \mathrm{GeV}$, mixing as measured by $\left\langle 0\left|G_{\mu}(x) J_{\nu}(y)\right| 0\right\rangle$, where $J$ is some quark current, is certainly small, $O\left(\alpha_{s}{ }^{3 / 2}\right)$. The 3.1 should be a good laboratory source of gluonic resonances, decaying through them into ordinary hadrons.
On the theoretical plausibility of well-defined bumps in $R_{G}$, we only comment that those models based on a quark-gluon Lagrangian that explicitly exhibit hadrons all include quarkless mesons. Some of these are dual vortex rings, ${ }^{34}$ box excitations in lattice gauge theories, ${ }^{35}$ and bags $^{36}$ containing only gluons. The last of these is particularly suggestive: Why shouldn't the gluonic spectrum resemble that of bags with massless quarks?

\section{ACKNOWLEDGMENTS}

We are indebted to many colleagues for help during the course of this work. Particular thanks are due to Leonard Susskind for discussions at the 1974 Aspen Institute where this work was begun and to Kenneth Wilson for his encouragement. At Harvard, we were continually questioned and helped by Helen Quinn, Howard Georgi, R. Shankar, Sheldon Glashow, Howard Schnitzer, Asim Yildiz, Karl Strauch, and Alvaro De Rújula. We also thank John Kogut, Harald Fritzsch, Peter Minkowski, David Gross, and Ivan Muzinich for helpful remarks.
*Alfred P. Sloan Fellow. Research sponsored in part by the U.S. Atomic Energy Commission Grant No. AT(11-1)-2220 and by the National Science Foundation Grant No. NSF GP-40397X, Harvard University.

$\dagger$ Junior Fellow, Harvard University Society of Fellows. Work supported in part by the U. S. Atomic Energy Commission Grant No. AT(11-1)-68.

${ }^{1}$ J. J. Aubert et al ., Phys. Rev. Lett. 33, 1404 (1974).

${ }^{2}$ J.-E. Augustin et al ., Phys. Rev. Lett. 33, 1406 (1974).

${ }^{3}$ G. S. Abrams et al., Phys. Rev. Lett. 33, 1453 (1974).

${ }^{4}$ J.-E. Augustin et al ., Phys. Rev. Lett. 34,764 (1975).

${ }^{5} \mathrm{~A}$ summary of an earlier stage of this work is given in

T. Appelquist and H. David Politzer, Phys. Rev. Lett. 34,43 (1975).

${ }^{6} \mathrm{See}$, for example, B. Richter, invited talk at the Conference on Lepton-Induced Reactions, Irvine, California, 1973 (unpublished).

${ }^{7}$ B. J. Bjorken and S. L. Glashow, Phys. Lett. 11, 225 (1964); S. L. Glashow, J. Iliopoulos, and L. Maiani, Phys. Rev. D 2, 1285 (1970).

${ }^{8}$ For a review of asymptotically free theories and their connection to Bjorken scaling, see H. David Politzer, Phys. Rep. 14C, 130 (1974).

${ }^{9}$ The large size of $m_{c}$ will not affect approximate leptoproduction scaling since charmed quarks are not present in ordinary hadrons.

${ }^{10} \mathrm{~W}$. A. Bardeen, H. Fritzsch, and M. Gell-Mann, in Scale and Conformal Invariance in Hadron Physics (Wiley, New York, 1973), p. 139.

${ }^{11}$ B. W. Lee and J. Zinn-Justin, Phys. Rev. D $\underline{5}, 3121$ (1972).

${ }^{12}$ M. Weinstein, Phys. Rev. D $\underline{7}, 1854$ (1973).
${ }^{13}$ M. Gell-Mann, R. J. Oakes, and B. Renner, Phys. Rev. 175,2195 (1968).

${ }^{14} \mathrm{~A}$ discussion of some aspects of this problem is given by T. Appelquist and J. Carazzone, Phys. Rev. D 11 , 2856 (1975).

${ }^{15} \mathrm{G}$. 't Hooft, Nucl. Phys. B61, 455 (1973).

${ }^{16}$ S. Weinberg, Phys. Rev. $\bar{D} \underline{8}, 3497$ (1973).

${ }^{17}$ T. Appelquist and H. Georgi, Phys. Rev. D 8,4000

(1973); A. Zee, Phys. Rev. D 8 , 4038 (1973).

${ }^{18}$ S. L. Adler, Phys. Rev. D 10, 3714 (1974).

${ }^{19}$ T. Kinoshita, J. Math. Phys. 3 , 650 (1962). This analysis did not explicitly include Yang-Mills theories. However, we have checked that no special problems arise in low orders of perturbation theory.

${ }^{20}$ The simplicity of this analysis results from two features special to $\sigma_{\text {tot }}$ in the single-photon approximation. First, it is a function of a single kinematic variable, so scale transformations have immediate physical significance. And second, to lowest order in $e$, there are no "anomalous dimensions," so Eq. (2.3) follows rigorously from the renormalization-group equation for the vacuum polarization. There is no problem in principle in using this equation for timelike momentum. The problems arise in using perturbation theory.

${ }^{21}$ R. Jost and J. M. Luttinger, Helv. Phys. Acta 23, 201 (1950); G. Källén and A. Sabry, K. Dan. Vidensk. Selsk. Mat.-Fys. Medd. 29 , no. 17 (1955).

${ }^{22} \mathrm{~J}$. Schwinger, Particles, Sources and Fields, Vol. II (Addison-Wesley, New York, 1973), Chapter 5-4.

${ }^{23} \mathrm{E}$. Eichten et al., Phys. Rev. Lett. 34, 369 (1975).

${ }^{24}$ B. Harrington, S. Y. Park, and A. Yildiz, Phys. Rev. Lett. 34 (1975); H. J. Schnitzer, Harvard report, 1974 
(unpublished); K. S. Jhung, K. H. Chung, and R. S. Willey, Univ, of Pittsburgh report, 1974 (unpublished). ${ }^{25}$ T. Appelquist, A. De Rújula, H. D. Politzer, and S. L. Glashow, Phys. Rev. Lett. 34, 365 (1974).

${ }^{26}$ M. K. Gaillard, B. W. Lee, and J. L. Rosner, Rev. Mod. Phys. 47, 277 (19́75).

${ }^{27} \mathrm{~T}$ wo important qualifying comments should be made about this computation and other similar ones in this section. The first and most serious concerns the use of only the minimal gluon contribution for $\left|M_{h}\right|^{2}$. The second concerns the roles of weak binding in (4.1) and (4.2).

A criticism of the minimal gluon mechanism is the following. If more gluons are added, the typical momentum going into each one is expected to go down and the effective coupling constant at each vertex should go up. Adding enough gluons might then make the theory strongly coupled and the perturbation expansion would break down. Whether this happens requires a careful analysis of diagrams which we have not yet carried out. However, the first part is very similar to the Kinoshita analysis (Ref. 19) which underlies the calculation of $\boldsymbol{R}(s)$ in the light-quark theory. The mass singularities associated with putting the gluons on mass shell are expected to cancel in each order since a total hadronic width is being calculated. Recall that the mass-shell singularities associated with the initial $c$ and $\bar{c}$ quarks have been factored into the amplitude $A$ (Fig. 5). This becomes the wave-function factor $\psi(0)$ in the weakbinding expression for the width. If mass singularities are absent, then the question of dividing up the total momentum remains. The only source of a large dimensional parameter that could lead to large logarithms and hence large effective couplings would be some fraction of the total mass corresponding to a multigluon division of the momentum. If this happens here, then it will also happen in the calculation of $R(s)$ in a light-quark theory or even in the calculation of a Euclidean Green's function. The entire use of asymptotic freedom is then in question.

Given the minimal gluon mechanism, what about binding corrections? These are just the relativistic corrections mentioned in the text. They enter in the flux factor since the annihilation is not exactly local and in the matrix element $\boldsymbol{M}_{\boldsymbol{h}}$ since it is not to be evaluated with the $c$ and $\bar{c}$ exactly at rest. All these effects are expected to be of order $v / c$ or equivalently $1 /\langle r\rangle \boldsymbol{m}_{c}$. Numerical estimates based on Ref. 23 suggest that they could amount to approximately $20 \%$ corrections to $\alpha_{s}$. This should be taken as the level of accuracy of Sec. IV.

${ }^{28} \mathrm{This}$ number is the width for the nonelectromagnetic decay into hadrons. See F. Gilman, invited talk presented at the Orbis Scientiae II, University of Miami, Coral Gables, Florida, 1975 (unpublished); D. R. Yennie, Phys. Rev. Lett. 34, 239 (1975).

${ }^{29}$ R. Dashen, I. J. Muzinich, B. W. Lee, and C. Quigg, Fermilab Report No. FERMILAB-Pub-75/18-THY, 1975 (unpublished).

${ }^{30}$ S. Okubo, Phys. Lett. 5, 165 (1963); G. Zweig, CERN Reports Nos. TH. 401, TH. 412, 1964 (unpublished); J. Iizuka, Prog. Theor. Phys. Suppl. 37-38, 21 (1966) .

${ }^{31}$ A. De Rújula and S. L. Glashow, Phys. Rev. Lett. 34, 46 (1975).

${ }^{32}$ J. D. Jackson, Lawrence Berkeley Laboratory, Report No. JDG/74-2, 1974 (unpublished).

${ }^{33}$ This description was suggested privately by H. Fritzsch. ${ }^{34} \mathrm{H}$. Nielson and P. Oleson, Nucl. Phys. B61, 45 (1972). ${ }^{35} \mathrm{~J}$. Kogut and L. Susskind, Phys. Rev. D 11, 395 (1975).

${ }^{36}$ A. Chodos, R. L. Jaffe, K. Johnson, C. B. Thorn, and V. F. Weiskopff, Phys. Rev. D 9 , 3471 (1974). 